\title{
A Low-Cost Pressure Sensor for Maternal Uterine Activity Monitoring
}

\author{
Han-Chang $\mathrm{Wu}^{1}$, Shuenn-Tsong Young ${ }^{2}$, and Te-Son Kuo ${ }^{1,3}$ \\ ${ }^{1}$ Department of Electrical Engineering, National Taiwan University, \\ Roosevelt Rd., Sec 4, Taipei 106, Taiwan, R. O. C., Email: wuman@tpts1.seed.net.tw \\ ${ }^{2}$ Institute of Biomedical Engineering, National Yang-Ming University, \\ No. 155, Sec. 2, Li-Nong St., Shih-Pai, Taipei 112, Taiwan, R.O.C \\ ${ }^{3}$ Graduate Institute of Biomedical Engineering, National Taiwan University, \\ Roosevelt Rd., Sec 4, Taipei 106, Taiwan, R. O. C.
}

\begin{abstract}
In this paper we presented a novel pressure sensor that is highly suitable for assessing the variation of uterine activity to prevent preterm labor of high-risk women. The rate of preterm labor is increasing in modern societies and it results in huge medical expenditures. However, preterm labor is hard to perceive in advance because the sensitivity and significance of self-examine is low. The home uterine activity monitoring (HUAM) is a new methodology, which automatically sense the uterine activity and then send the information to physicians or practitioners for further advising or medication. The HUAM can effectively prevent the preterm labor, but the conventional pressure sensors are uncomfortable and expensive. Our new design is based on a Colpitts self-oscillating circuit and a ferrite coil. It senses the pressure of uterine contraction by the coil displacement, which changes the output frequency. The proposed pressure sensor is light-weighted, stable, lowcost, and is appropriate for portable and long-term applications of uterine monitoring.
\end{abstract}

\section{Introduction}

Labor occurring between 20 and 37 weeks of gestation is called preterm labor. Preterm labor is the major reason that causes high perinatal morbidity and mortality and thus results in huge expenditures for premature infant care [1]. Statistical data shows that the rate of preterm labor lies in $8 \sim 10 \%$ and costs over three billion dollars each year in the United States 2]. Several schemes for early diagnosis are conducted to prevent preterm labor, such as cervical seeking and self-palpating of uterine contractions [3]. The risk of preterm birth could be reduced significantly with prompt treatment of tocolytic medication. However, it is difficult for the mother-to-be to sense the symptom of preterm labor because the sense of contractions is confusing. Claudia et al. [4] showed that only $17 \%$ of uterine contractions are accurately perceived. In the mid 1980s, a new methodology known as home uterine activity monitoring (HUAM) was proposed to detect the uterine contractions automatically [5]. Many clinical studies have been conducted and the HUAM method was concluded with positive results to prevent preterm labor [5-8] for high-risk women. In the HUAM system, the uterine contractions were monitored by an external pressure sensor (usually named tocodynamometer) strapped on maternal upper abdomens. The uterine contractions were then recorded and transmitted to practitioners by telephones and modems for treatment advises. The practitioner evaluates the received magnitude and frequency of the uterine contractions. If the contraction frequency exceeds a threshold, the women will be asked to take the HUAM one more time or to have a doctor immediately.

The HUAM system consists of a pressure sensor, a recorder, and a transmitting device. The tocodynamometer, which senses the pressure variation of the uterine, is the most critical component. It was conventionally implemented by strain gages, linear variable differential transformers (LVDT), or piezoelectric materials [9-10]. These sensors were complicated and more efforts were necessary for system calibration.

In this paper, we proposed a novel pressure sensor that can transfer the pressure signal of uterine contractions into frequency variations by a ferrite core and a selfoscillating circuit. Our design has the advantages including simple, stable, reliable, low power dissipation, and low cost. This new pressure sensor is highly suitable for portable devices in long-term ambulatory monitoring applications [11]. 


\section{System Design}

The structure of the proposed sensor is illustrated in figure 1. Uterine contractions are transduced to vertical displacements of a ferrite core equipped with four appropriate springs according to the Hooke's Law. A selfoscillating Colpitts circuit then converts the displacements into frequency variations. As the schematic illustrated in figure 2 , the frequency of the oscillator is determined by a transistor $\left(Q_{1}\right)$, two capacitors $\left(C_{1}, C_{2}\right)$, and a coil $\left(L_{1}\right)$ from the following expression:

$$
f=\frac{1}{2 \pi \sqrt{L_{1}\left(\frac{C_{1} C_{2}}{C_{1}+C_{2}}\right)}}
$$

The displacement of the ferrite core determines the inductance of the coil and thus alternates the frequency of the Colpitts oscillator. A buffer circuit $\left(Q_{2}\right)$ is designed to isolate the Colpitts oscillator from unwanted capacitance from connectors and cables. The output signal of the oscillator is linked to a portable monitor [11] and shaped to TTL level by another buffer circuit $\left(Q_{3}\right)$. The output frequency is counted by a microcontroller. The microcontroller then scales the frequencies to $0 \sim 100$ units for further processing. Because the conversion from displacement to frequency output is nonlinear in (1), the microcontroller also maps the nonlinear frequencies to linear output. A power management circuit $\left(Q_{4}\right)$ is also included.

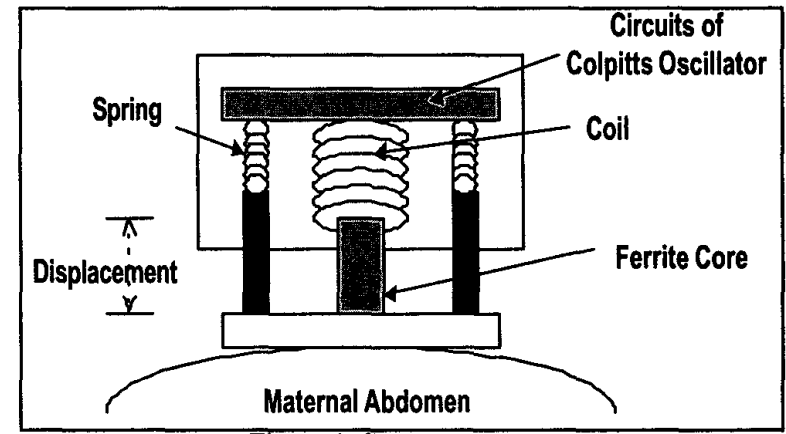

Figure 1. Sensor structure.

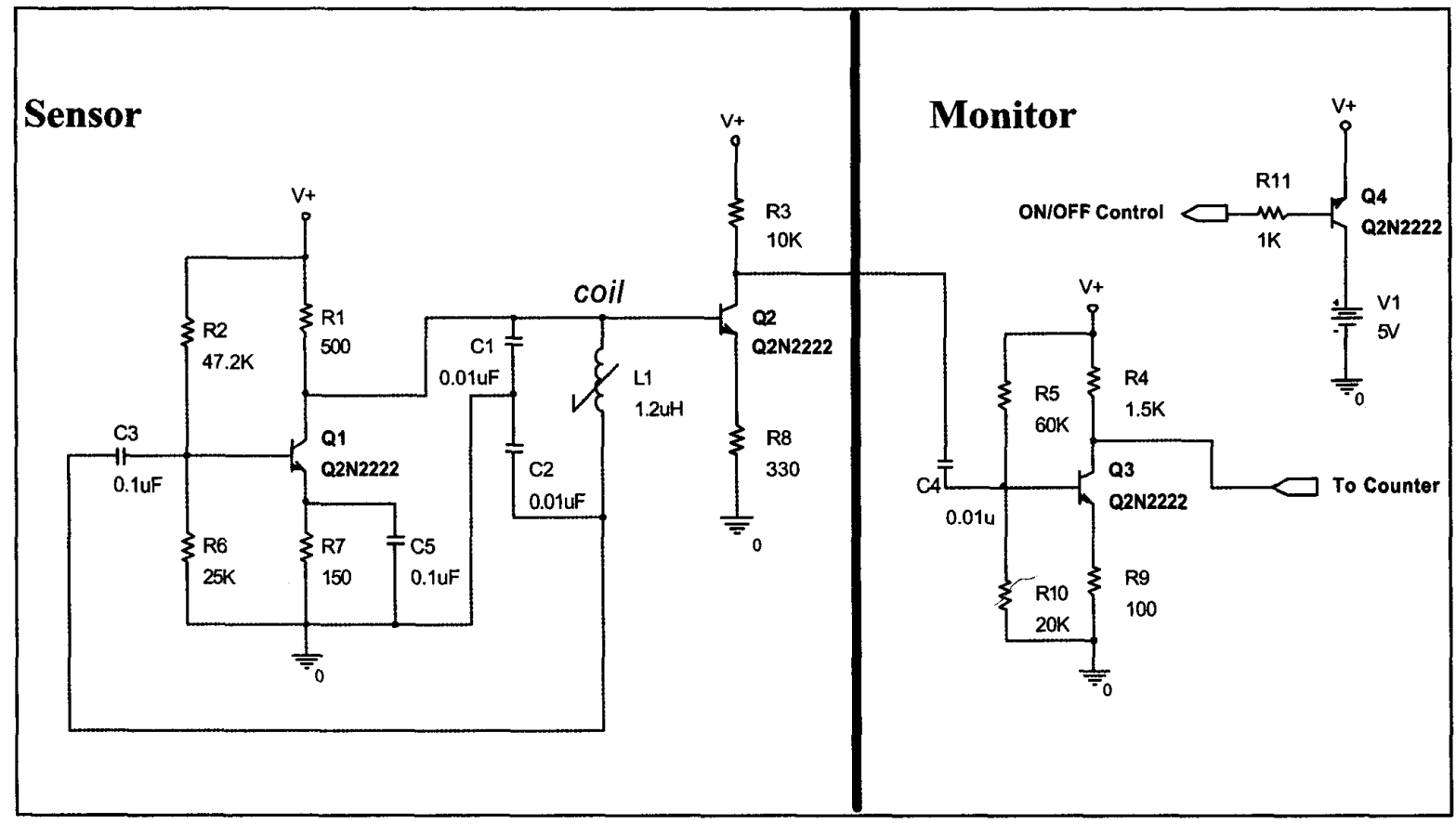

Figure 2. Schematic of the sensor. 


\section{Results}

The specifications of the proposed sensor are summarized in table 1 . This sensor could be operated with $3 \mathrm{~V} \sim 10 \mathrm{~V}$ voltage supply. Its frequency variation is less than $0.1 \%$ working at tempature 0 to $45^{\circ} \mathrm{C}$. The oscillator becomes stable within 500us after turning on the power. The outline of the sensor is shown in figure 3 .

\begin{tabular}{cc}
\hline \hline Size & $70 \mathrm{~mm} * 40 \mathrm{~mm} * 33 \mathrm{~mm}$ \\
\hline Weight & $34 \mathrm{~g}$ \\
\hline Power Consumption & $8.2 \mathrm{~mA} \mathrm{@} 5 \mathrm{~V}$ input \\
\hline Stable Time & $<500 \mathrm{us}$ \\
\hline Frequency Variation & $<0.1 \%$ \\
\hline Output Range & $0 \sim 100$ Units \\
\hline \hline
\end{tabular}

Table 1. System specifications.

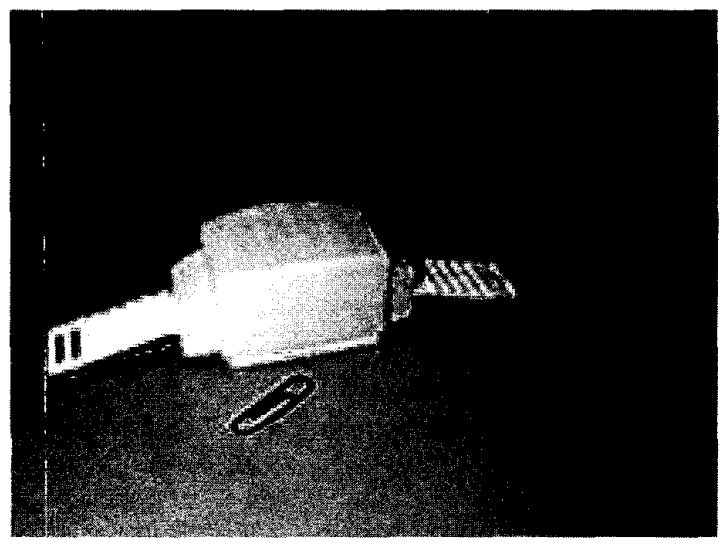

Figure 3. Sensor outline.

\section{Discussions and Conclusions}

The major concerns of the HUAM is the inconvenience to wear the sensor of the uterine contractions. The sensor is also related to the reliability and cost of the HUAM. Compared to current systems, our proposed sensor has smaller size and lighter weight and it thus reduces the maternal loading. The sensor output is stable and reliable under varient environments. The calibration can be easily achieved by software. Furthurmore, 24-hour monitoring of uterine contraction is possible with its low power dissipation. Therefore, a lowcost, high performance, and convenient HUAM system could be accomplished with the proposed sensor.

\section{References}

[1] Mary L. Moore and Margaret C. Freda, "Reducing preterm and low birthweight births: still a nursing challenge, " American Journal of Maternal Child Nursing vol. 23, pp.200-208, July, 1998.

[2] David G. Weismiller, "Preterm Labor," American Family Physician, pp. 593-604, February, 1999.

[3] "Home uterine activity monitoring for preterm labor: review article," Journal of the American Medical Association, vol. 270, pp.371-376, 1993.

[4] Claudia A. Beckmann, Charles R.B. Beckmann, Gary J. Stanziano, Niki K. Bergauer, and Chester B. Martin, "Accuracy of maternal perception of preterm uterine activity," American Journal of Obstetrics \& Gynecology, vol. 174, pp. 672-675, February, 1996.

[5] Katz M, Gill PJ, and Newman RB, "detection of preterm labor by ambulatory monitoring of uterine activity: a preliminary report," Obstetrics \& Gynecologu, vol. 68, pp. 773-778, December, 1986.

[6] Ronald J.Wapner, David B. Cotton, Raul Artal, Ronald J. Librizzi, and Michael G. Ross, "A randomized multicenter trial assessing a home uterine activity monitoring device used in the absence of daily nursing contact," American Journal of Obstetrics \& Gynecology, vol. 172, pp. 10261034, March, 1995.

[7] Theodore Colton, Herbert Kayne, Yuqing Zhang, and Timothy Heeren, "A metaanalysis of home uterine activity monitoring," American Journal of Obstetrics \& Gynecology, no. 173, pp. 1499-1505, November, 1995.

[8] Michael J. Corwin, Susan M. Mou, Shirazali G. sunderji, Stanley Gall, Helen How, Vinu Patel, and Mark Gray, "Multicenter randomized clinical trial of home uterine activity monitoring: Pregancy outcomes for all women randomized," American Journal of Obstetrics \& Gynecology, vol. 175, pp. 1281-1285, November, 1996.

[9] David G. Tweed and Eric LaWhite, "Tocodynamometer," U.S. Patent No. 3,945,373, 1976.

[10] Philip O. Isaacson, "Tocodynamometer," U.S. Patent No. 4,640,295, 1987.

[11] Chih-Ling Lin, Han-Chang Wu, Tz-Ti Liu, Maw-Huei Lee, Te-Son Kuo, and Shuenn-Tsing Young, " A Portable Monitor for Fetal Heart Rate and Uterine Contraction," IEEE Engineering in Medicine and Biology, November,1997. 